Conclusions Plasma endothelins level and ratio of thromboxane $\mathrm{B}_{2}$ to 6-Keto- $\mathrm{PGF}_{\mathrm{la}}$ increased in the patients with primary hyperlipidaemia. Xuezhikang not only effectively adjusted blood lipids level but also reduced plasma endothelins level and ratio of thromboxane $\mathrm{B}_{2}$ to 6-Keto-PGFla.

\section{e0670 THE POTENTIAL ROLE OF SERUM CYSTATIN C FOR RENAL FUNCTION EVALUATION OF VERY OLD PATIENTS WITH CHRONIC KIDNEY DISEASE}

doi:10.1136/hrt.2010.208967.670

Yang Yong, Cheng Qingli. Chinese Pla General Hospital

Objective To evaluate the potential role of Serum Cystatin C (Scys) for glomerular filtration rate (GFR) of very old patients with chronic kidney disease (CKD).

Methods 50 male CKD patients who were above 75 years old in our hospital were selected for detecting GFR by nephrogram using ${ }^{99 \mathrm{~m} T c-D T P A}$. Meanwhile, the concentrations of serum creatinine (Scr) and Scys were tested. eGFR were calculated by the equations of Cockcroft-Gault (CG), simplified Chinese Modification of Diet in Renal Disease equations for Scr, and the equations of Hoek, Le Bricon for Scys respectively. The results were analysed statistically. Results All patients were diagnosed to CKD stage 3 to stage 5 according to the results of GFR by nephrogram. The concentration of Scys was not effected by age, weight and height ( $p>0.05)$, and the average concentration of Scys had significant difference among stage 3,4 , and 5 of CKD patients $(\mathrm{p}<0.05)$. For Scys and the equations of Hoek and Le Bricon, their correlation coefficients to GFR were higher than those of Scr and the equations of CG, Chinese simplified Modification of Diet in Renal Disease in stage 5 CKD patients, but lower in stage 3 and 4 CKD patients $(p<0.05)$.

Conclusions The evaluation accuracy of GFR using Scys were not concordant in different stage of very old CKD patients. Whether Scys was the better marker for evaluating GFR of very old CKD patients needed future researching.

\section{e0671 THE THERAPEUTIC EFFECTS OF COMPLEX DANSHEN ZHUSHEYE TO THE RENAL DYSFUNCTION AFTER NEONATAL ASPHYXIA}

doi:10.1136/hrt.2010.208967.671

Fang Xiaoyi, Lin Niyang, Li Yuguang. First Affiliated Hospital of Shantou University Medical College

Objective To explore the therapeutic effects of complex Danshen Zhusheye to the renal dysfunction after neonatal asphyxia.

Methods To collect the clinical data of 80 cases of neonatal renal dysfunction after asphyxia and divide them into Danshen group and control group randomly. The complex Danshen Zhusheye was used in the Danshen group with the dose of $4 \mathrm{ml}$ in term infants and $2 \mathrm{ml}$ in preterm infants, intravenous drip, once per day, from day 3 after birth till day 10 after birth. The other treatments in two groups were similar. The urine outputs were recorded from the first day after birth till the day when urine outputs were normal. Blood urea nitrogen (BUN) and serum creatinine (SCr) were detected at day 3 and day 10.

Results The differences of urine outputs, BUN and SCr in two groups at day 3 were not significant. At day 10, compared with day 3 , the urine outputs were increased and the BUN and SCr were decreased in both groups. The urine outputs were $(2.61 \pm 1.05) \mathrm{ml} /$ $\mathrm{kg} \cdot \mathrm{h}^{-1}$ and $(2.50 \pm 1.12) \mathrm{ml} / \mathrm{kg} \cdot \mathrm{h}^{-1}, \mathrm{BUN}$ were $(3.35 \pm 1.12) \mathrm{mmol} / \mathrm{l}$ and $(6.55 \pm 2.21) \mathrm{mmol} / \mathrm{l}$ and SCr were $(66.51 \pm 8.11) \mu \mathrm{mol} / \mathrm{l}$ and $(100.31 \pm 8.98) \mu \mathrm{mol} / 1$ in Danshen group and control group respec- tively at day 10. Compared with the control group, the BUN and SCr were decreased in Danshen group with $p<0.01$ at day 10 . The day that the urine outputs were normal was $5.02 \pm 1.00(3 \sim 7)$ days in Danshen group and $7.12 \pm 2.11(5 \sim 10)$ days in control group. The difference was highly significant with $\mathrm{p}<0.01$.

Conclusion The recover time was earlier with Danshen treatment in neonatal renal dysfunction after asphyxia, which can hint that the complex Danshen Zhusheye is effective to protect the renal function after neonatal asphyxia.

\section{e0672 TREATMENT OF ATHEROSCLEROTIC RENAL ARTERY STENOSIS INVOLVING RENAL ARTERY BIFURCATIONS}

doi:10.1136/hrt.2010.208967.672

Zheng Bin, Yan Hongbing. Beijing Anzhen Hospital

Objective To investigate the efficacy of kissing stent or kissing balloon technique for renal artery bifurcation with atherosclerotic renal artery stenosis.

Methods There were five patients included, who were with atherosclerotic renal artery stenosis involving renal artery bifurcation.

Results The reference vascular diameter of main renal artery was $5.6 \pm 0.4 \mathrm{~mm}$. The reference vascular diameter of renal artery distal to bifurcation were $3.4 \pm 0.4 \mathrm{~mm}$ and $3.6 \pm 0.5 \mathrm{~mm}$. Kissing balloon technique was used in three patients with obvious residual stenosis, and kissing stent technique was used in two patients without obvious residual stenosis. Blood pressure was lowered in two patients, unchanged in three patients. Serum creatine was lowered in one patient.

Conclusion As for angiographic residual stenosis, it seems that kissing stent technique is more efficacious than kissing balloon technique.

\section{e0673 STENT IMPLANTATION BEFORE CARDIAC SURGERY WITH CARDIOPULMONARY BYPASS HAS NO EFFECT ON IMPAIRED RENAL FUNCTION IN PATIENTS WITH RENAL ARTERY STENOSIS}

doi:10.1136/hrt.2010.208967.673

Zheng Bin, Yan Hongbing. Beijing Anzhen Hospital

Objective To investigate whether stent implantation before cardiac surgery with cardiopulmonary bypass (CPB) have any effect on the development of impaired renal function in patients with renal artery stenosis (RAS).

Methods In this retrospective study, 69 patients with RAS were included, among whom there were 38 patient receiving renal artery stent implantations just before CPB. To assess acute kidney injury (AKI) after $\mathrm{CPB}$, serum urea nitrogen (SUN), serum creatinine ( $\mathrm{SCr}$ ), creatinine clearance $(\mathrm{CrCl})$ were recorded at baseline, at the end of operation, during the 1 st and 2 nd postoperative $24 \mathrm{~h}$. Patients with abnormal SCr $(>106 \mu \mathrm{mol} / \mathrm{l})$ before cardiac surgery were not included in this study.

Results Baseline characteristics were similar between groups. Changes of SUN, SCr and $\mathrm{CrCl}$ were similar between groups. The incidences of AKI $(22.6 \%, 26.3 \%)$ in patients without or with stent implantation were not significantly deferent from each other. In patients without stent implantation, AKI defined by RIFLE occurred in $7(22.6 \%)$ patients: $5(16.1 \%)$ with class R, 2 (6.5\%) with I, and no patients with $\mathrm{F}$. In patients with stent implantation, 10 patients (26.3\%) had an episode of AKI during hospitalisation: 6 (15.8\%) had RIFLE-R, 4 (10.5\%) had RIFLE-I, and no patients had RIFLE-F.

Conclusion There is no data suggesting that stent implantation can improve the renal dysfunction after CPB. However, it cannot be concluded that RAS is not associated with AKI after CPB. 